\section{The role of red cell distribution width in the differential diagnosis of iron deficiency anemia and non-transfusion- dependent thalassemia patients}

\author{
Pokpong Piriyakhuntorn, \\ Adisak Tantiworawit, \\ Thanawat Rattanathammethee, \\ Chatree Chai-Adisaksopha, \\ Ekarat Rattarittamrong, \\ Lalita Norasetthada
}

Division of Hematology, Department of Internal Medicine, Faculty of Medicine, Chiang Mai University, Chiang Mai, Thailand

\begin{abstract}
This study aims to find the cut-off value and diagnostic accuracy of the use of RDW as initial investigation in enabling the differentiation between IDA and NTDT patients. Patients with microcytic anemia were enrolled in the training set and used to plot a receiving operating characteristics (ROC) curve to obtain the cut-off value of RDW. A second set of patients were included in the validation set and used to analyze the diagnostic accuracy. We recruited 94 IDA and 64 NTDT patients into the training set. The area under the curve of the ROC in the training set was 0.803 . The best cut-off value of RDW in the diagnosis of NTDT was $>21.0 \%$ with a sensitivity and specificity of $81.3 \%$ and $55.3 \%$ respectively. In the validation set, there were 34 IDA and 58 NTDT patients using the cut-off value of $21.0 \%$ to validate. The sensitivity, specificity, positive predictive value and negative predictive value were $84.5 \%, 70.6 \%, 83.1 \%$ and $72.7 \%$ respectively. We can therefore conclude that RDW $>21.0 \%$ is useful in differentiating between IDA and NTDT patients with high diagnostic accuracy.
\end{abstract}

\section{Introduction}

Microcytic anemia is the most common form of anemia seen in medical practice. ${ }^{1}$ Both iron deficiency anemia (IDA) and thalassemia are common causes of microcytic anemia in Thailand. ${ }^{2}$ Thalassemia has variety phenotypes, which can be divided into thalassemia trait, which is asymptomatic, and thalassemia disease. The essential factor in distinguishing the severity of thalassemia disease is transfusion-dependence.
Transfusion-dependent thalassemia (TDT) patients have obvious clinical features such as severe anemia, a thalassemic face, growth retardation, massive hepatosplenomegaly and require regular blood transfusion; therefore, the diagnosis can be fairly conclusive before further investigation. Patients with non-transfusion-dependent thalassemia (NTDT) do not require lifelong regular transfusion for survival, but may require occasional transfusions in certain settings. Undiagnosed NTDT patients, who have never had a transfusion, may present with microcytic anemia without any gross thalassemic features, which can mimic the characteristics of IDA patients. Decisions regarding further investigations can be challenging. A definite diagnosis of these two conditions can be made easily by hemoglobin typing and iron studies but they may take time, are costly and also are not available in some areas. Red cell distribution width values (RDW), one of the red blood cell indices, which will already have been reported in the complete blood count $(\mathrm{CBC})$, reflects the degree of anisocytosis of red blood cells. This value has been used to differentiate between patients with IDA and a thalassemia trait for decades. ${ }^{3,4}$ From previous studies, varied cut-off values from $13.4 \%$ to $21.0 \%$ were used and gave a wide range of sensitivity and specificity. $2,5-16$ The cut-off values allowing differentiation between IDA and NTDT and the diagnostic accuracy of this differentiation have never been properly studied. This study will identify the particular cut-off value of RDW for the differentiation between IDA and NTDT to facilitate the giving of proper initial management and inform further investigations before a definite diagnosis is made.

\section{Materials and Methods}

The study was conducted at the Department of Internal Medicine at Maharaj Nakorn Chiang Mai Hospital, Chiang Mai University. All adult patients with microcytic anemia, both outpatients and inpatients, attending the Department of Internal Medicine in the period March 2011 to August 2016 were enrolled. Data collected retrospectively using the electronic medical record system were age, sex, current and past medical illness, history of blood transfusion and laboratory data $(\mathrm{CBC}$, serum creatinine, iron studies, hemoglobin typing). Inclusion criteria were a hemoglobin count of less than $12.0 \mathrm{~g} / \mathrm{dL}$ for women and $13.0 \mathrm{~g} / \mathrm{dL}$ for men, a mean corpuscular volume (MCV) below $80 \mathrm{fL}$, and meeting
Correspondence: Adisak Tantiworawit, Division of Hematology, Department of Internal Medicine, Faculty of Medicine, Chiang Mai University, 110 Intravaroros road, A. Muang, Chiang Mai, Thailand 50200. Tel.: 6653935482 - Fax: 6653935481.

E-mail: adisak.tan@cmu.ac.th; atantiwo@yahoo.com

Key words: Red cell distribution width; microcytic anemia; iron deficiency anemia; non-transfusion-dependent thalassemia.

Acknowledgements: we would like to thank Ms. Antika Wongthani, Head of the Analytical \& Statistical Data Unit, Research Institute for Health Sciences, Chiang Mai University most sincerely for her suggestions regarding the statistics in this study. P.P. designed the research, collected, summarized, analyzed the clinical data and wrote the paper; A.T. designed the research, obtained the research grant, analyzed the data, wrote the paper, give critical comment and corresponding author; T.R., E.R., C.C., L.N. revised the manuscript. This study was supported by a research grant from the Faculty of Medicine, Chiang Mai University.

Funding: this study was supported by a research grant from the Faculty of Medicine, Chiang Mai University.

Contributions: PP designed the research, collected, summarized, analyzed the clinical data and wrote the paper; AT designed the research, obtained the research grant, analyzed the data, wrote the paper, give critical comment and corresponding author; TR, ER, CC, LN revised the manuscript.

Conflict of interest: the authors declare no conflict of interest.

Ethical approval: this study was approved by the ethical research committee, Faculty of Medicine, Chiang Mai University.

Received for publication: 23 January 2018. Accepted for publication: 29 June 2018.

This work is licensed under a Creative Commons Attribution-NonCommercial 4.0 International License (CC BY-NC 4.0).

CCopyright P. Piriyakhuntorn et al., 2018 Licensee PAGEPress, Italy

Hematology Reports 2018; 10:7605

doi:10.4081/hr.2018.7605

the diagnostic criteria of either IDA or NTDT. Exclusion criteria included: i) none completion of both iron studies and hemoglobin typing; ii) IDA with concomitant thalassemia trait or disease; iii) diagnosed chronic kidney disease according to the definition in Kidney Disease: Improving 
Global Outcomes 2012 (KDIGO); iv) splenectomized; v) transfusion-dependence or history of blood transfusion in past 3 months; vi) pregnancy.

The diagnosis of IDA was made by transferrin saturation $<16 \%$ or/and serum ferritin $<30 \mathrm{ng} / \mathrm{mL}$. The diagnosis of NTDT was made from recorded clinical data and compatible hemoglobin typing result by high performance liquid chromatography (HPLC).

The patients were divided into 2 groups according to the date of the $\mathrm{CBC}$ test, the two groups being the training set and the validation set. The training set, consisted of patients recruited between March 2011 and August 2015, and their data was used to plot a receiving operating characteristics (ROC) curve to obtain the cut-off value of RDW. The validation set, consisted of patients recruited between September 2015 and August 2016, and was used to analyze the accuracy of diagnosis.

The sample size formula for estimating the sensitivity of the test ${ }^{17}$ was used to calculate the basis for a significance level at 0.05 , a $95 \%$ confidence interval, with anticipated sensitivity at $80 \%$ and $10 \%$ allowable error margins in estimating specificity.
The sample size of the training set was at least 62 subjects in each arm.

Complete blood counts and determination of red cell parameters were performed using automated hematology analyzers, Siemens ADVIA ${ }^{\circledR} 2120$ which are calibrated for standardization of results every 6 months.

IBM SPSS statistics version 23.0 was used for the statistical analysis of the results. The receiver operating characteristic (ROC) curve analysis was also used to demonstrate the diagnostic performance of RDW. P values of $\leq 0.05$, calculated using a Chi-square test was used to compare categorical variables and Independent samples t-test was used to compare between continuous variables, were considered significant. STATA $^{\circledR}$ version 14.0 was used for the analysis of diagnostic accuracy and 95\% confidence interval in the validation set.

\section{Results}

We enrolled total 1773 microcytic anemia patients between March 2011 and August 2016. 1523 patients were excluded; the remaining 250 patients were included in the study (Figure 1). The training set consists of 94 IDA patients $(59 \%)$ and 64 NTDT patients (41\%). The baseline characteristics of both groups were mostly statistically similar. The exception was the proportion of females in the IDA patients is greater (79.8\% vs 54.7\%, $\mathrm{p}=0.001$ ) (Table 1). The mean age of the training set was 49.96 years. The mean hemoglobin level was 8.26 $\mathrm{g} / \mathrm{dL}$. The majority of the population in the study had a moderate to severe degree of anemia, based on the WHO definition. The mean MCV was $64.75 \mathrm{fL}$. The mean RDW was significantly higher in NTDT patients $(24.52 \pm 4.09$ vs $20.09 \pm 3.52$, p $<0.001)$. The ROC curve of RDW used in the diagnosis of NTDT had an area under the curve of 0.803 (Figure 2). The best RDW cut-off value obtained from the ROC curve in the diagnosis of NTDT was more than $21.0 \%$ with a sensitivity and specificity of $81.3 \%$ and $55.3 \%$ respectively. The other nearby cut-off values and their given sensitivity and specificity are shown in Table 2 .

During a 1-year period, from September 2015 to August 2016, the validation set consisted of 34 IDA patients $(37 \%)$ and 58 NTDT patients $(63 \%)$. The baseline charac-

Table 1. Baseline characteristics of the study subjects.

\begin{tabular}{|c|c|c|c|c|c|c|}
\hline \multirow[t]{2}{*}{ Parameters } & \multicolumn{2}{|c|}{ Training set } & \multicolumn{4}{|c|}{ Validation set } \\
\hline & $\begin{array}{c}\text { IDA } \\
(n=94)\end{array}$ & $\begin{array}{l}\text { NIDT } \\
(n=64)\end{array}$ & P value & $\begin{array}{c}\text { IDA } \\
(n=34)\end{array}$ & $\begin{array}{l}\text { NIDT } \\
(\mathrm{n}=58)\end{array}$ & P value \\
\hline Sex Female (n, \%) & $75(79.8)$ & $35(54.7)$ & 0.01 & $29(85.3)$ & $38(65.5)$ & 0.04 \\
\hline Age (year, mean \pm SD) & $47.97 \pm 18.17$ & $52.88 \pm 16.98$ & 0.09 & $47.21 \pm 22.52$ & $49.28 \pm 16.11$ & 0.64 \\
\hline $\begin{array}{l}\text { Hemoglobin }(\mathrm{g} / \mathrm{dL}, \text { mean } \pm \mathrm{SD}) \\
\geq 11.0 \\
8.0-10.9 \\
<8.0\end{array}$ & $\begin{array}{l}8.26 \pm 1.80 \\
4(4.3) \\
50(53.2) \\
40(42.6)\end{array}$ & $\begin{array}{l}8.25 \pm 1.20 \\
0(0.0) \\
39(60.9) \\
25(39.1)\end{array}$ & 0.97 & $\begin{array}{l}7.94 \pm 1.76 \\
2(5.9) \\
13(38.2) \\
19(55.9)\end{array}$ & $\begin{array}{l}8.43 \pm 1.24 \\
3(5.2) \\
38(65.5) \\
17(29.3)\end{array}$ & 0.16 \\
\hline Mean corpuscular volume (fL, mean \pm SD) & $64.92 \pm 7.92$ & $64.49 \pm 6.79$ & 0.72 & $63.57 \pm 8.46$ & $66.42 \pm 7.20$ & 0.09 \\
\hline $\begin{array}{l}\text { Red cell distribution width }(\%, \text { mean } \pm \mathrm{SD}) \\
\mathrm{Hb} \geq 11.0 \\
\mathrm{Hb} 8.0-10.9 \\
\mathrm{Hb}<8.0\end{array}$ & $\begin{array}{l}20.09 \pm 3.52 \\
14.92 \pm 2.75 \\
19.76 \pm 3.80 \\
21.03 \pm 2.68\end{array}$ & $\begin{array}{c}24.52 \pm 4.09 \\
- \\
23.50 \pm 3.51 \\
26.11 \pm 4.49\end{array}$ & $<0.01$ & $\begin{array}{l}19.79 \pm 3.36 \\
14.35 \pm 0.49 \\
18.90 \pm 2.79 \\
20.97 \pm 3.19\end{array}$ & $\begin{array}{l}24.55 \pm 3.62 \\
21.33 \pm 4.75 \\
24.62 \pm 3.86 \\
24.97 \pm 2.72\end{array}$ & $<0.01$ \\
\hline Ferritin (mg/dL, median (IQR) & $\begin{array}{c}9.00 \\
(5.00-18.75)\end{array}$ & $\begin{array}{c}889.00 \\
(494.00-1591.00)\end{array}$ & $<0.01$ & $\begin{array}{c}10.50 \\
(4.00-17.50)\end{array}$ & $\begin{array}{c}962.50 \\
(583.50-1556.75)\end{array}$ & 0.01 \\
\hline Transferrin saturation (\%, median (IQR) & $\begin{array}{c}3.69 \\
(2.50-5.83)\end{array}$ & $\begin{array}{c}31.90 \\
(24.10-57.55)\end{array}$ & $<0.01$ & $5.67 \pm 3.84$ & $\begin{array}{c}24.87 \\
(19.84-47.78)\end{array}$ & $<0.01$ \\
\hline Serum creatinine (g/dL, mean \pm SD) & $0.78 \pm 0.21$ & $0.72 \pm 0.21$ & 0.07 & $0.75 \pm 0.20$ & $0.71 \pm 0.21$ & 0.43 \\
\hline $\begin{array}{l}\text { Thalassemia type (n, \%) } \\
\alpha \text {-thalassemia } \\
\mathrm{Hb} \mathrm{H} \\
\mathrm{Hb} \mathrm{H} \text { with CS } \\
\mathrm{Hb} \mathrm{H} \text { with Hb E trait } \\
\beta \text {-thalassemia } \\
\beta^{0} \text {-thal/Hb E disease } \\
\beta^{+} \text {-thal/Hb E disease } \\
\text { Homozygous Hb E }\end{array}$ & $\begin{array}{c}51(79.7) \\
43 \\
5 \\
3 \\
13(20.3) \\
2 \\
11 \\
0 \\
\end{array}$ & & & $\begin{array}{c}41(70.7) \\
34 \\
6 \\
1 \\
17(29.3) \\
10 \\
6 \\
1 \\
\end{array}$ & & \\
\hline
\end{tabular}

IDA, iron deficiency anemia; NTDT, non-transfusion-dependent thalassemia; Hb, hemoglobin level (g/dL); CS, Constant Spring; SD, standard deviation. 
teristics of both groups were also statistically similar apart from the proportion of female patients $(85.3 \%$ vs $65.5 \%, \mathrm{p}=0.04)$, the same trend as in the training set. The mean RDW was also significantly higher in NTDT patients $(24.55 \pm 3.62$ vs $19.79 \pm$ $3.36, \mathrm{p}<0.001)$. The sensitivity, specificity, positive predictive value and negative predictive value in the diagnosis of NTDT by RDW $\geq 21.0 \%$ in the validation set were $84.5 \%, 70.6 \%, 83.1 \%$ and $72.7 \%$ respectively (Table 3 ).

The proportion of the thalassemia phenotype in NTDT patients among the training and validation sets were $79.7 \%$ and $70.7 \%$ for alpha-thalassemia respectively, and $20.3 \%$ and $29.3 \%$ for beta-thalassemia (Table 1).

\section{Discussion}

Distinguishing IDA from thalassemia has been and still is an ongoing problem in Thailand, where the prevalence of thalassemia is high. ${ }^{18}$ Until now, many studies have shown a high efficacy of RDW in distinguishing IDA from the thalassemia trait, but this has not been the same for thalassemia disease. ${ }^{2,5-16}$ Our study used RDW for differentiating between IDA and NTDT in adults with moderate to severe microcytic anemia, according to the WHO classification. ${ }^{19}$ At a cut-off value of $21.0 \%$, it gives high diagnostic accuracy, especially up to a sensitivity value of $84.5 \%$, in the diagnosis of NTDT.

Iron overload is a common complication among thalassemia patients, leading to many serious co-morbidities. Not only transfusion-dependent thalassemia patients suffer from this condition, it can also occur in NTDT patients who have not been transfused due to an increased intestinal absorp- tion of iron. Iron supplements could be harmful to these patients. ${ }^{20}$ So, in our study, we have chosen a cut-off value which gives us a high sensitivity for the diagnosis of NTDT, while the specificity value is not too low, in order to minimize the false negative rate and avoid the prescription of iron supplements to undiagnosed NTDT patients as an empirical treatment before a definite

Table 2. RDW cut-off values and their sensitivity and specificity in the diagnosis of NTDT in the training set.

\begin{tabular}{lcc} 
RDW cut-off value & Sensitivity & Specificity \\
$20.5 \%$ & $87.5 \%$ & $50.0 \%$ \\
$21.0 \%$ & $81.3 \%$ & $55.3 \%$ \\
\hline $21.5 \%$ & $76.6 \%$ & $68.1 \%$ \\
\hline
\end{tabular}

RDW, red cell distribution width; NTDT, non-transfusion-dependent thalassemia.

Table 3. Diagnostic accuracy of diagnosis of NTDT by RDW $\geq 21.0 \%$ in the validation set.

\begin{tabular}{lcc}
\hline Parameters & Value & $95 \%$ Confidence interval \\
Sensitivity & $84.5 \%$ & $72.6-92.7$ \\
Specificity & $70.6 \%$ & $52.5-84.9$ \\
\hline Positive predictive value & $83.1 \%$ & $71.0-91.6$ \\
Negative predictive value & $72.7 \%$ & $54.5-86.7$ \\
\hline Positive likelihood ratio & 2.87 & $1.69-4.89$ \\
Negative likelihood ratio & 0.22 & $0.12-0.42$ \\
\hline Accuracy & $77.5 \%$ & $68.5-86.6$ \\
\hline
\end{tabular}

RDW, red cell distribution width; NTDT, non-transfusion-dependent thalassemia.

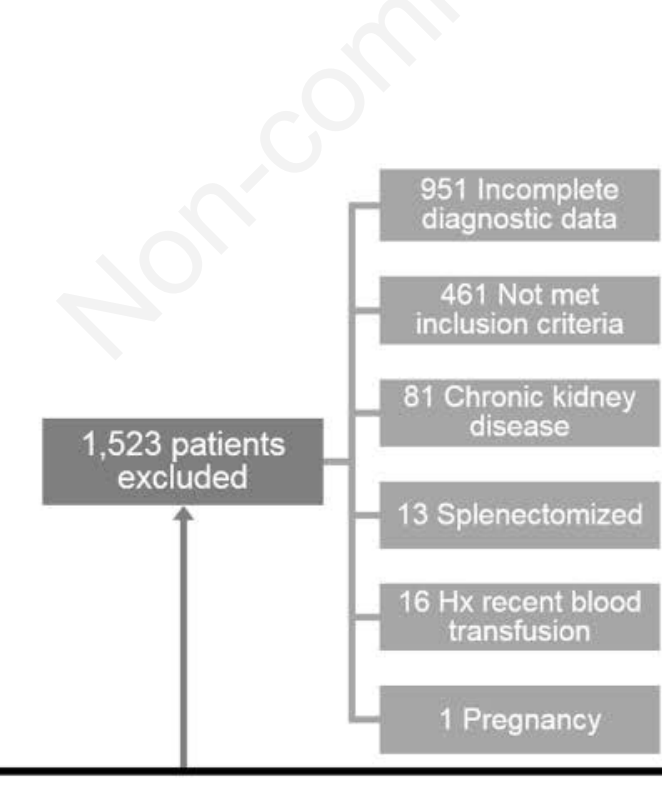

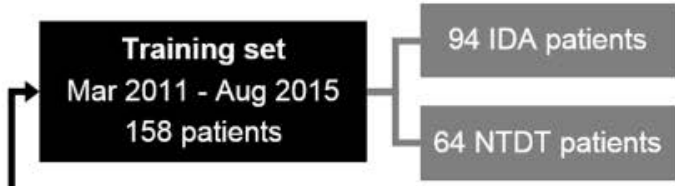

Validation set Sep 2015 - Aug 2016 92 patients
34 IDA patients

58 NTDT patients

Figure 1. Study population flow. 
diagnosis can be made.

A recent study by Johannes J.M.L. Hoffmann et al., ${ }^{16}$ the first meta-analysis of $\mathrm{RBC}$ indices for distinguishing between the thalassemia trait and IDA in patients with microcytic anemia, reported diagnostic accuracy from RDW using data collected in 48 studies worldwide, including a total of 12,039 subjects. With a cut-off value at $15 \%$, the sensitivity and specificity were $62 \%$ and $68 \%$ with an Area under the curve (AUC) of 0.778 . The specificity is comparable to our study but our sensitivity is higher. This may lead to the conclusion that RDW is more suitable for differentiating patients with a greater severity of thalassemia syndrome, like NTDT, in our study from those with iron deficiency anemia. Likewise, D. Viswanath et al. ${ }^{10}$ studied the diagnostic accuracy of an abnormally high RDW in the diagnosis of IDA in various grades and reported the greater the severity of anemia the patients had, the greater the sensitivity of RDW when used for the diagnosis of IDA. Lima et al. ${ }^{9}$ chose a RDW cut-off value of $21.0 \%$, giving a sensitivity of $90 \%$ and specificity $77 \%$ in distinguishing IDA from the beta thalassemia trait. Previous studies were mainly using RDW and other $\mathrm{RBC}$ indices to distinguish IDA from thalassemia trait, in which iron overload does not normally occur, even when the patient is receiving iron supplements. Thus, researchers may not need to include this and choose a cut-off value, which gives a high sensitivity in the diagnosis of IDA.

New generations of automated CBC machines also provide data concerning a new interesting value, specifically the percentage of microcytic red cells with a volume less than $60 \mathrm{fl}$ (\%Micro R). A value of more than $20 \%$ discriminates the beta thalassemia trait from mild IDA with $93.7 \%$ sensitivity and $75.4 \%$ specificity (AUC $0.938,95 \%$ CI $0.903-0.964) .{ }^{21}$ This simple value adds a higher level of diagnostic accuracy to our RDW value but the data was collected only in cases of mild severity of iron deficiency anemia and thalassemia syndrome, so its applicability in Thailand where greater severity of IDA and thalassemia is quite common needs to be considered.

Limitations of this study include, first, only pure IDA and pure NTDT were included in the study. Concomitant IDA and thalassemia (including traits), which can be occasionally found in Thailand where prevalence of the thalassemia trait is high, ${ }^{18}$ were excluded. Other causes of normocytic anemia such as pregnancy and chronic kidney disease were also excluded. This may overestimate the diagnostic accuracy and the real-world effectiveness should be examined further. Second, this is a retrospective study. The data were reviewed totally from electronic medical records. There was some incomplete data, including blood transfusion history, which may influence the results. Third, 951 out of 1773 patients $(53.6 \%)$ were excluded due to incomplete laboratory diagnostic data. This may be considered as selection bias. Fourth,

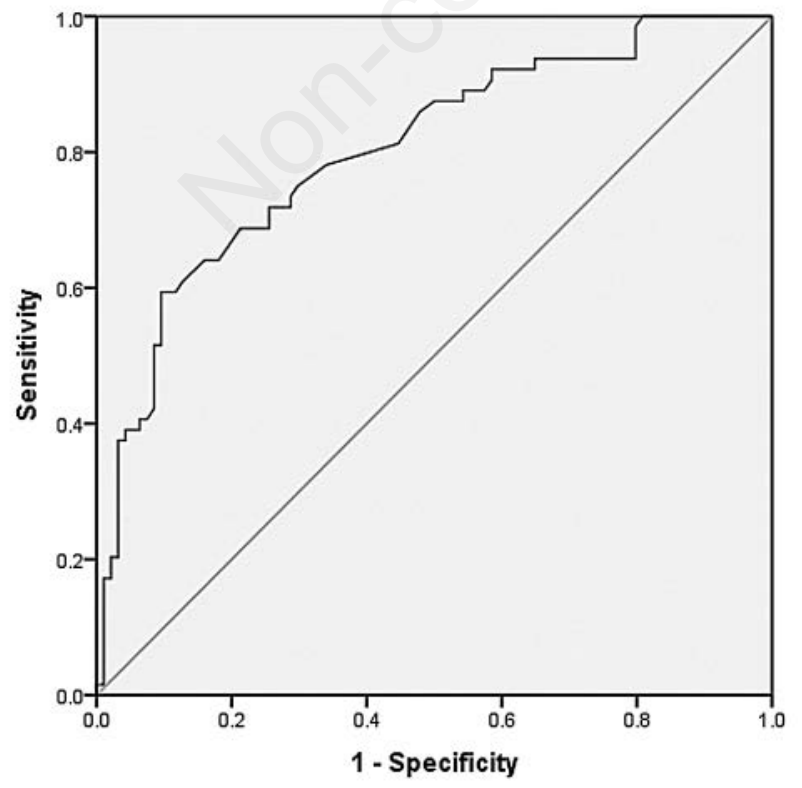

Figure 2. ROC curve of diagnosis of NTDT by RDW from the training set. RDW, red cell distribution width; NTDT, non-transfusion-dependent thalassemia; ROC, receiving operating characteristics.

the baseline characteristics in both groups are not statistically equal. The proportion of females in the IDA groups of both the training and the validation sets were higher than in the NTDT groups. But this might not have an effect a lot because prevalence of IDA in females is more than males in general. ${ }^{1}$ Finally, our study included mainly moderate to severe anemia groups. This may affect using RDW at this cut-off value to differentiate the causes in a mild anemia group. But from a subgroup analysis of mild anemia in the validation set, the mean RDW of IDA was $14.35 \%$ while the mean RDW of NTDT was $21.33 \%$, so the cut-off value of $21.0 \%$ may be reasonably applicable but this would need further confirmation in a large number of these patients.

In the future, we suggest further research to enable the development of an algorithm for use in the evaluation of microcytic anemia patients. This would need to include other basic laboratory results in addition to the RDW value to increase the diagnostic power of RDW in the diagnosis of NTDT. This is challenging but it may be very useful in developing countries where budgets are limited.

\section{Conclusions}

From our study, a RDW more than $21.0 \%$ may be useful in enabling differentiation between IDA and NTDT patients with a high level of diagnostic accuracy. This value can help to provide a provisional diagnosis, give information for appropriate further investigation and guide empirical treatment before a definitive diagnosis is made.

\section{References}

1. Bruno de Benoist EM, Ines Egli, Mary Cogswell, eds. Worldwide prevalence of anaemia 1993-2005. Geneva: WHO global database on anaemia; 2008.

2. Plengsuree $S$, Punyamung M, Yanola J, et al. Red cell indices and formulas used in differentiation of beta-thalassemia trait from iron deficiency in thai adults. Hemoglobin 2015;39:235-9.

3. Bessman JD, Gilmer PR, Jr., Gardner FH. Improved classification of anemias by MCV and RDW. Am J Clin Pathol 1983;80:322-6.

4. Aslan D, Gumruk F, Gurgey A, Altay C. Importance of RDW value in differential diagnosis of hypochrome anemias. Am J Hematol 2002;69:31-3.

5. Flynn MM, Reppun TS, Bhagavan NV. 
Limitations of red blood cell distribution width ( $\mathrm{rdw}$ ) in evaluation of microcytosis. Am J Clin Pathol 1986; 85:445-9.

6. Thompson WG, Meola T, Lipkin M, Jr., Freedman ML. Red cell distribution width, mean corpuscular volume, and transferrin saturation in the diagnosis of iron deficiency. Arch Intern Med 1988;148:2128-30.

7. van Zeben D, Bieger R, van Wermeskerken RK, et al. Evaluation of microcytosis using serum ferritin and red blood cell distribution width. Eur J Haematol 1990;44:106-9.

8. Kim SK, Cheong WS, Jun YH, et al. Red blood cell indices and iron status according to feeding practices in infants and young children. Acta Paediatr 1996;85:139-44.

9. Lima CS, Reis AR, Grotto HZ, et al. Comparison of red cell distribution width and a red cell discriminant function incorporating volume dispersion for distinguishing iron deficiency from beta thalassemia trait in patients with microcytosis. Sao Paulo Med J 1996;114:1265-9.

10. Viswanath D, Hegde R, Murthy V, et al. Red cell distribution width in the diagnosis of iron deficiency anemia.
Indian J Pediatr 2001;68:1117-9.

11. AlFadhli SM, Al-Awadhi AM, AlKhaldi D. Validity assessment of nine discriminant functions used for the differentiation between iron deficiency anemia and thalassemia minor. J Trop Pediatr 2007;53:93-7.

12. Ntaios G, Chatzinikolaou A, Saouli Z, et al. Discrimination indices as screening tests for beta-thalassemic trait. Ann Hematol 2007;86:487-91.

13. Aulakh R, Sohi I, Singh T, Kakkar N. Red cell distribution width ( $\mathrm{rdw}$ ) in the diagnosis of iron deficiency with microcytic hypochromic anemia. Indian J Pediatr 2009;76:265-8.

14. Ng EH, Leung JH, Lau YS, Ma ES. Evaluation of the new red cell parameters on beckman coulter dxh800 in distinguishing iron deficiency anaemia from thalassaemia trait. Int $\mathrm{J}$ Lab Hematol 2015;37:199-207.

15. Zaghloul A, Al-Bukhari TA, Bajuaifer $\mathrm{N}$, et al. Introduction of new formulas and evaluation of the previous red blood cell indices and formulas in the differentiation between beta thalassemia trait and iron deficiency anemia in the makkah region. Hematology 2016;21:351-8.

16. Hoffmann JJ, Urrechaga E, Aguirre U.
Discriminant indices for distinguishing thalassemia and iron deficiency in patients with microcytic anemia: A meta-analysis. Clin Chem Lab Med 2015;53:1883-94.

17. Kumar R, Indrayan A. Receiver operating characteristic (roc) curve for medical researchers. Indian Pediatr 2011;48:277-87.

18. Fucharoen S, Winichagoon P. Hemoglobinopathies in southeast Asia. Hemoglobin 1987;11:65-88.

19. World Health Organization. Haemoglobin concentrations for the diagnosis of anaemia and assessment of severity [Internet]. Vitamin and Mineral Nutrition Information System, (WHO/NMH/NHD/MNM/11.1); 2011 [cited 12 Feb 2017]. Available from: http://www.who.int/vmnis/indicators/ha emoglobin.pdf

20. Peters M, Heijboer H, Smiers F, Giordano PC. Diagnosis and management of thalassaemia. BMJ 2012;344:e228.

21. Urrechaga E, Borque L, Escanero JF. Erythrocyte and reticulocyte parameters in iron deficiency and thalassemia. J Clin Lab Anal 2011;25:223-8. 